\title{
Pleomorphic archaeal viruses: the family Pleolipoviridae is expanding by seven new species
}

\author{
Tatiana A. Demina ${ }^{1}$ (D) Hanna M. Oksanen ${ }^{1}$ (D)
}

Published online: 24 June 2020

(c) The Author(s) 2020

\begin{abstract}
Established in 2016, the family Pleolipoviridae comprises globally distributed archaeal viruses that produce pleomorphic particles. Pseudo-spherical enveloped virions of pleolipoviruses are membrane vesicles carrying a nucleic acid cargo. The cargo can be either a single-stranded or double-stranded DNA molecule, making this group the first family introduced in the $10^{\text {th }}$ Report on Virus Taxonomy including both single-stranded and double-stranded DNA viruses. The length of the genomes is approximately 7-17 kilobase pairs, or kilonucleotides in the case of single-stranded molecules. The genomes are circular single-stranded DNA, circular double-stranded DNA, or linear double-stranded DNA molecules. Currently, eight virus species and seven proposed species are classified in three genera: Alphapleolipovirus (five species), Betapleolipovirus (nine species), and Gammapleolipovirus (one species). Here, we summarize the updated taxonomy of the family Pleolipoviridae to reflect recent advances in this field, with the focus on seven newly proposed species in the genus Betapleolipovirus: Betapleolipovirus HHPV3, HHPV4, HRPV9, HRPV10, HRPV11, HRPV12, and SNJ2.
\end{abstract}

\section{Introduction}

Archaeal viruses have unique morphological and genetic features as well as prominent roles in global ecological processes $[14,26]$. Among viruses infecting halophilic euryarchaeotes, pleomorphic ones represent the second largest group after tailed icosahedral viruses [14, 26]. Currently, a decade after the discovery of the first pleomorphic archaeal virus, Halorubrum pleomorphic virus 1 (HRPV-1), in 2009 [21], 18 such virus isolates are known [4, 5, 9, 17, 19]. Here, following the accumulation of new data, descriptions of new pleolipovirus isolates, and the recent submission of taxonomical proposals [10, 20], we provide an updated overview

This article is related to taxonomic proposals that have been approved by the ICTV Executive Committee and were awaiting ICTV ratification at the time of submission. The taxonomy proposed here may differ from any new taxonomy that is ultimately approved by the ICTV and is presented for discussion only but has no official standing.

Handling Editor: Sead Sabanadzovic.

Hanna M. Oksanen

hanna.oksanen@helsinki.fi

1 Molecular and Integrative Biosciences Research Programme, Faculty of Biological and Environmental Sciences,

University of Helsinki, Helsinki, Finland of this group of viruses, describing their life cycle, host range, virion components, and genomic content.

\section{The family Pleolipoviridae}

Pleomorphic archaeal viruses have been isolated from various hypersaline environments all over the world (Table 1, Fig. 1). The currently described pleolipoviruses have been isolated on halophilic archaeal strains belonging to the genera Halorubrum, Haloarcula, Halogeometricum, and Natrinema of the class Halobacteria. Pleolipoviruses establish non-lytic infection, presumably using budding as an exit mechanism. Virus particles are produced continuously, and the infection may slightly retard host growth (e.g., Halogeometricum pleomorphic virus 1 [HGPV-1]) [23] or impair it significantly (e.g., His2) [30]. As shown for His2, despite the growth retardation, host membranes remain intact during viral egress, as no ATP leakage or lowered oxygen consumption has been observed in infected cells [30]. Saline Natrinema sp. J7-1 virus 2 (SNJ2) is the only temperate pleolipovirus that has been described. The host range of pleolipoviruses is typically very narrow, often limited to the specific isolation host (Table 1). Some pleolipoviruses have been shown to tolerate a wide range of $\mathrm{NaCl}$ concentrations, 


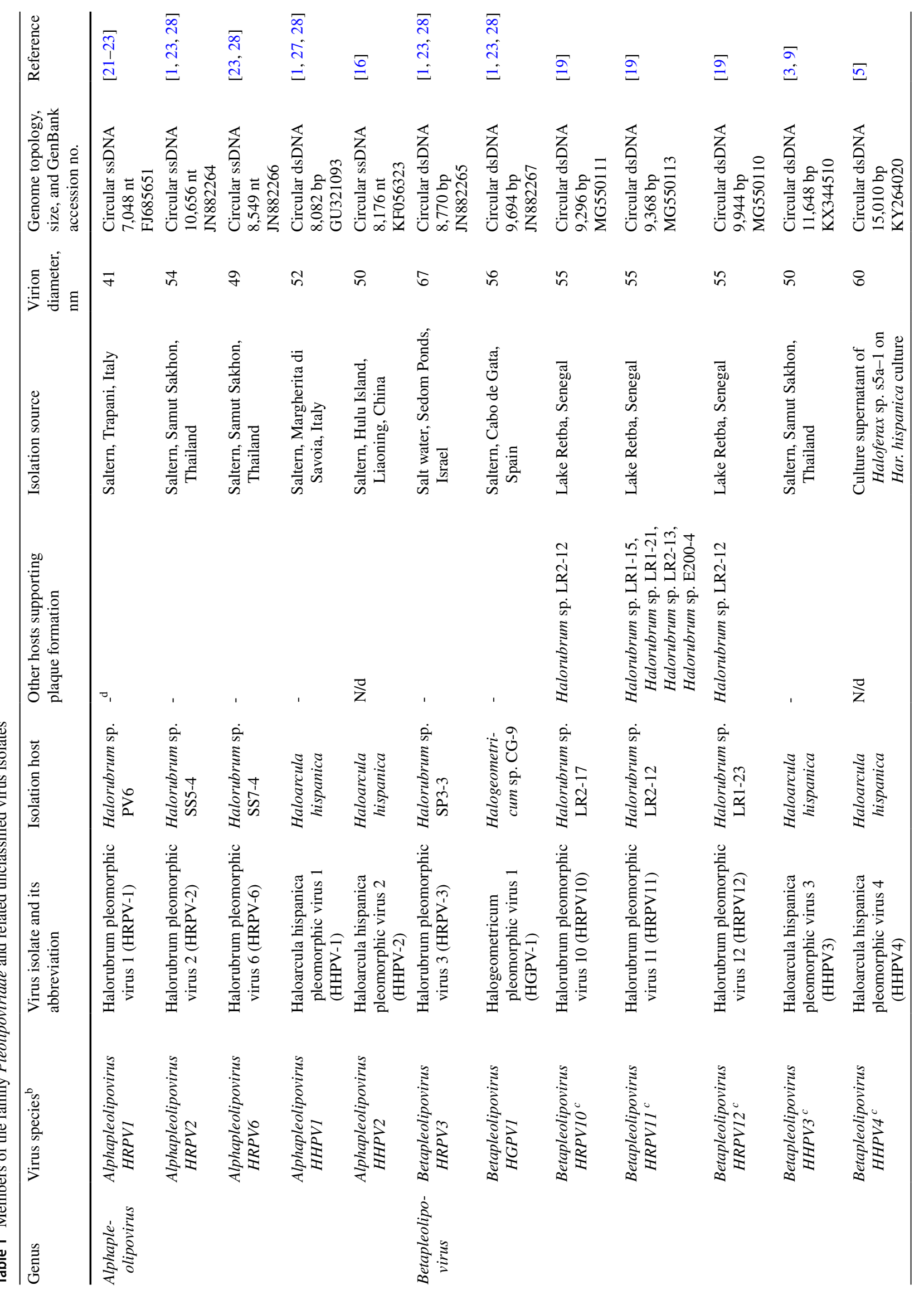




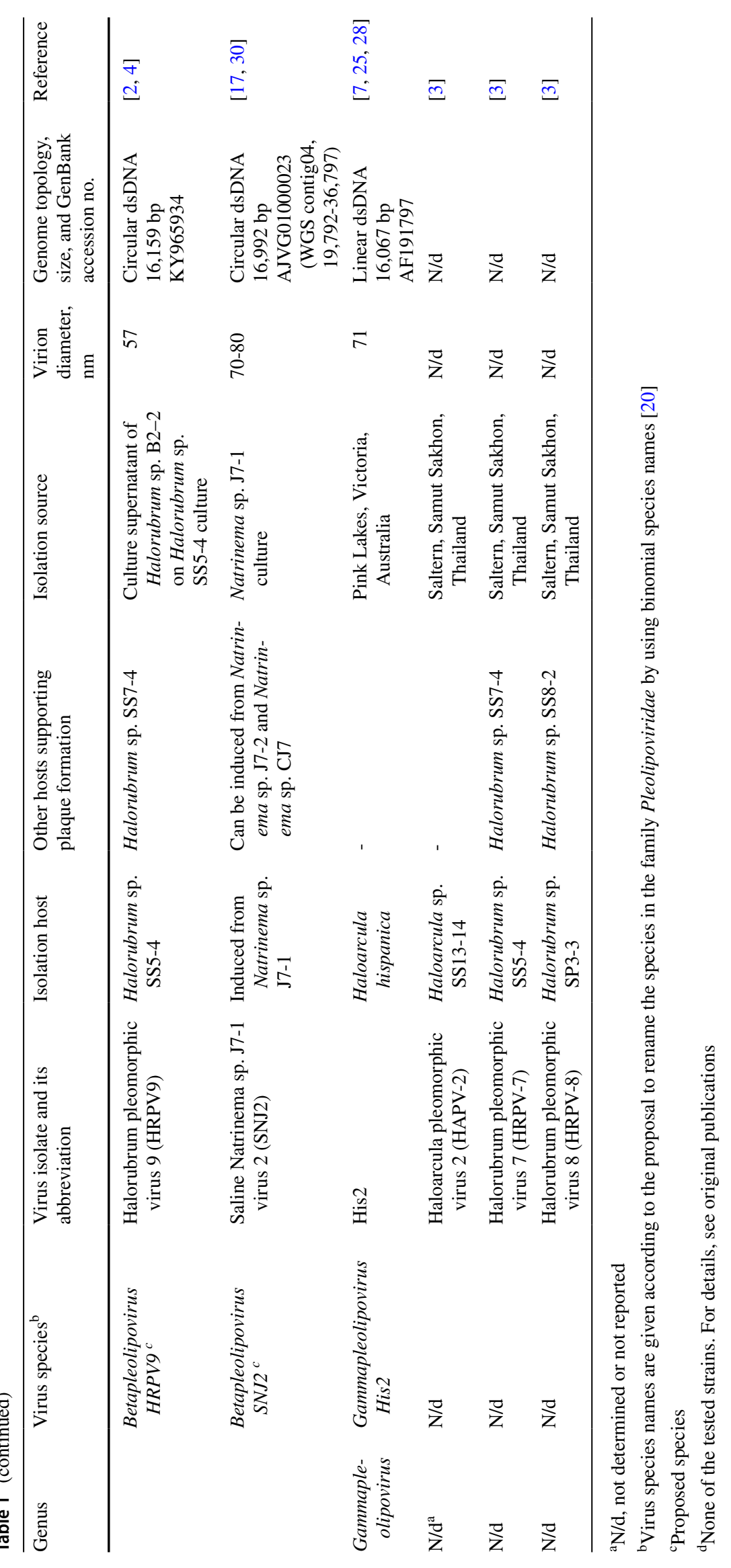


while others are more sensitive to lowered levels of $\mathrm{NaCl}$ or other ions [9, 23].

Pleolipoviruses lack a rigid protein capsid. Instead, their virions are simple, spherical, and flexible membrane vesicles of 40-80 nm in diameter [4-6, 9, 16, 17, 19, 23, 24] (Fig. 2). They have only 2-4 major structural protein types, either located at the inner side of the membrane or irregularly distributed on the virion surface as spikes (Fig. 2) [4, $5,9,17,19,23]$. Lipids forming the viral membrane vesicle are obtained non-selectively from the host membrane during virion assembly [4-6, 9, 17, 19, 23, 24]. Recent crystallographic structures of Halorubrum pleomorphic virus 2 (HRPV-2) and Halorubrum pleomorphic virus 6 (HRPV6) spike proteins showed that the monomeric pleolipoviral spike proteins undergo conformational changes and induce the fusion of the viral and host membranes. The spike protein is composed of two roughly equal domains, and its socalled V-shape represents a unique fold. The pre-fusion form of the spike protein is extended to an elongated conformation while being inserted into the membrane to initiate fusion [12].

Pleolipoviruses have circular single-stranded (ss) or double-stranded (ds) DNA genomes, except His2, whose genome is a linear dsDNA molecule (Table 1). Genome length ranges from $\sim 7$ to $\sim 17$ kilobase pairs or kilonucleotides. The dsDNA genomes of viruses belonging to the genus Betapleolipovirus may be interrupted by singlestranded regions, whose function is unclear $[5,9,17,19$, 28]. All pleolipovirus genomes carry a conserved block of collinear genes, encoding structural proteins, a putative NTPase, and other putative proteins (Fig. 2) [4, 19, 28]. The sequence identity between pleolipovirus genomes is typically low, with the following exceptions: The overall nucleotide sequence identity between Haloarcula hispanica pleomorphic virus 1 (HHPV-1) and Haloarcula hispanica pleomorphic virus 2 (HHPV-2) is $71 \%$ [16], as is the case with HRPV-2 and HRPV-6 [28]. Halorubrum pleomorphic virus 10 (HRPV10), Halorubrum pleomorphic virus 11 (HRPV11), and Halorubrum pleomorphic virus 12 (HRPV12), recently isolated from Lake Retba, also have highly similar genomes with $92-95 \%$ nucleotide sequence identity [19]. Furthermore, Haloarcula hispanica pleomorphic virus 3 (HHPV3) and Haloarcula hispanica pleomorphic virus 4 (HHPV4) share identical genomic regions (Fig. 3) [5]. In addition, pleolipovirus-related proviral regions commonly found in the haloarchaeal genomes may share up to $100 \%$ identity with viral genes $[4,7-9,11,17$, 19, 21, 27, 28]. Integrase genes are present in the HHPV4, SNJ2, and Halorubrum pleomorphic virus 9 (HRPV9) genomes (see below) $[4,5,17]$.

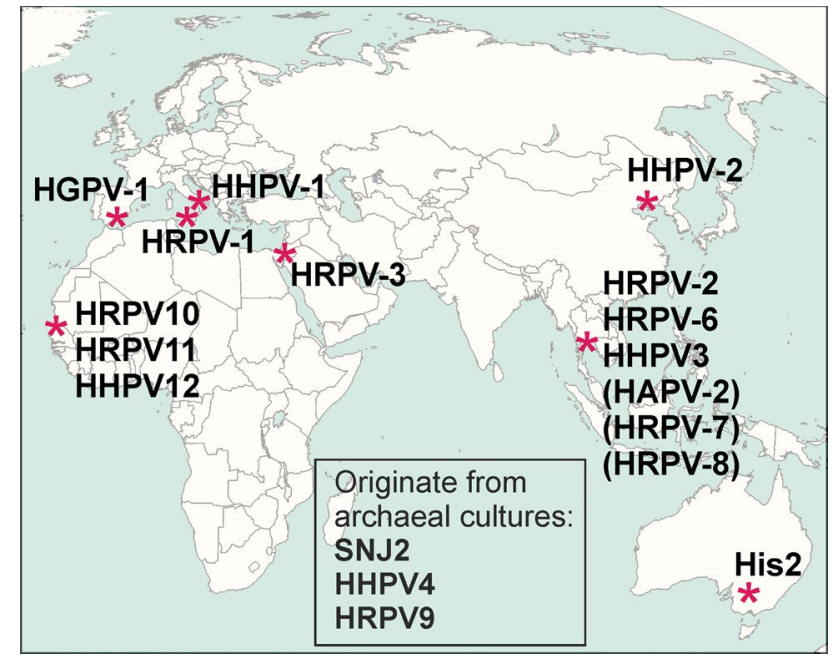

Fig. 1 Sites from which pleolipoviruses have been isolated (see also Table 1). Haloarcula hispanica pleomorphic virus 4 (HHPV4), Halorubrum pleomorphic virus 9 (HRPV9), and Saline Natrinema sp. J7-1 virus 2 (SNJ2) originate from archaeal cultures. Haloarcula pleomorphic virus 2 (HAPV-2), Halorubrum pleomorphic virus 7 (HRPV-7), and Halorubrum pleomorphic virus 8 (HRPV-8) shown in brackets are currently unclassified. Original map source: Wikimedia Commons

\section{Current taxonomy of the family Pleolipoviridae}

The family Pleolipoviridae currently has three genera containing fifteen species either approved (8 species) or newly proposed (7 species). The genera, Alpha-, Beta-, and Gammapleolipovirus, are distinguished based on the wholegenome sequence identity and gene content of their members (Table 1, Fig. 3) [6]. In addition to the conserved block of genes shared by all pleolipoviruses, the members of the three genera have specific open reading frames (ORFs). Alphapleolipoviruses encode a putative rolling-circle replication initiation protein, while the only described gammapleolipovirus, His2, encodes a putative protein-primed DNA polymerase, suggesting rolling-circle and protein-primed replication modes, respectively [28]. The linear dsDNA genome of His2 also has inverted terminal repeats and terminal proteins attached at the ends [7,25]. Betapleolipoviruses have two additional conserved ORFs downstream of the conserved block not found in other pleolipoviruses [4, 19, 28]. One of these (HGPV-1 ORF14 homologue), is suggested to be a replication initiation protein $[14,19]$. The genomes of three related pleolipoviruses, Haloarcula pleomorphic virus 2 (HAPV-2), Halorubrum pleomorphic virus 7 (HRPV-7), and Halorubrum pleomorphic virus 8 (HRPV-8) [3], have not been sequenced yet and therefore remain unclassified [6]. 

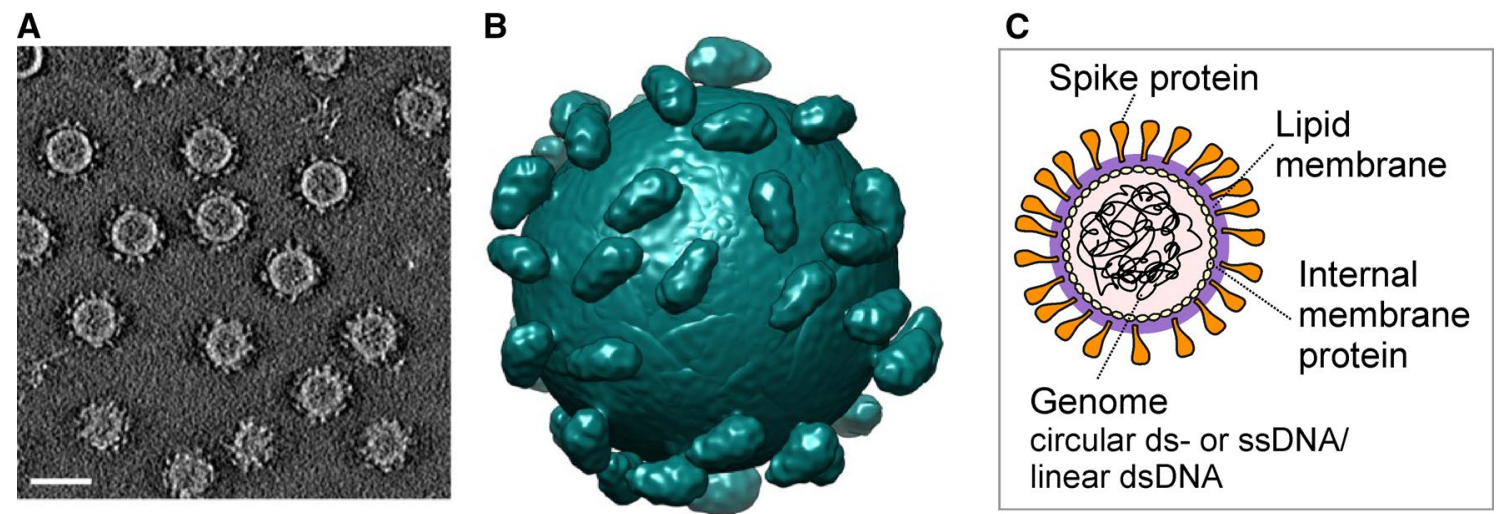

Fig. 2 Pleolipoviral virion. (A) Cryo-electron tomography of Halorubrum pleomorphic virus 6 (HRPV-6). A slice through a tomogram of HRPV-6. The bar is $40 \mathrm{~nm}$. (B) Structure of HRPV-6 virion based on

\section{Proposed taxonomic changes in the family Pleolipoviridae}

The proposals to create seven new species in the genus Betapleolipovirus and to change pleolipovirus species names using binomial names consisting of the genus name and the specific epithet have been approved recently by the ICTV Executive Committee [10, 20]. The newly proposed seven species are Betapleolipovirus HHPV3, HHPV4, HRPV9, HRPV10, HRPV11, HRPV12, and SNJ2 (Table 1). Nucleotide sequence identity of $\leq 95 \%$ has been proposed to be the criterion for demarcation of species in the genera of the family Pleolipoviridae [10]. The viruses representing the new species and species already in the family Pleolipoviridae have collinear dsDNA genomes with $\leq 95 \%$ nucleotide sequence identity shared between two genomes. The genomes of the seven new isolates carry the conserved block of genes and ORFs typical for the members of the family Pleolipoviridae, as well as two ORFs specific to the members of genus Betapleolipovirus [4, 5, 9, 17, 19].

\section{SNJ2 is the first described temperate pleolipovirus}

SNJ2 is the only pleolipovirus described to date for which a temperate life cycle has been demonstrated [17], although there are also other pleolipoviruses with integrase and phiH1-like repressor genes. SNJ2 can be induced from its host strain Natrinema sp. J7-1 [17] and is the only pleomorphic virus with Natrinema archaea as a host. In the provirus state, the SNJ2 genome is integrated into the host tRNA ${ }^{\text {Met }}$ gene, from which it is excised upon the induction of a lytic cycle. The host range of SNJ2 is very narrow, as it is known to infect only Natrinema sp. J7 derivatives, which differ by their plasmids (Table 1). The highest production of SNJ2 the tomography. (C) Schematic overview of pleolipoviral virion. Sections A and B are reproduced from reference 12

is observed from Natrinema sp. J7-1, which contains the plasmid pHH205, which is a proviral form of the icosahedral internal membrane-containing dsDNA virus SNJ1 (family Sphaerolipoviridae), suggesting that SNJ1 promotes the production of SNJ2 [17]. The pleomorphic SNJ2 virions are about $70 \times 80 \mathrm{~nm}$ in size and contain two major structural protein species. The SNJ2 lipid composition is similar to that of its host, except that SNJ2 seems to lack phosphatidylglycerophosphate methyl ester, which is present in Natrinema sp. J7-1, albeit in low abundance [17].

The SNJ2 genome is a circular dsDNA molecule with single-stranded interruptions associated with a conserved 'GCCCA' DNA motif, although the motif is not always followed by the interruption [17]. The same motif has been shown to precede single-stranded interruptions in the genome of the betapleolipovirus Halorubrum pleomorphic virus 3 (HRPV-3) [28]. The SNJ2 genome is 16,992 bp long, has a GC content of $59.1 \%$, and contains 25 ORFs. A conserved cluster of pleolipoviral genes is present in SNJ2, including ORFs specific to the genus Betapleolipovirus (Fig. 3). SNJ2 was the first pleolipovirus known to carry an integrase and a phiH1-like repressor gene in its genome [17]. The SNJ2 repressor has been suggested to function similarly to the repressor originally described in the halophilic archaeal tailed icosahedral virus phiH1, where it is involved in lytic-lysogenic life cycle switches [17, 29]. The SNJ2 integrase excises the virus genome from the host chromosome, but for integration, two small accessory proteins are also needed $[17,31]$. The SNJ2-type integrases are widely found in archaeal genomes, being associated with proviruses and other mobile elements $[17,31]$. These integrases are suggested to form a distinct family within the tyrosine recombinase superfamily, having five invariant active site residues of the $\mathrm{R}_{\mathrm{I}} \ldots \mathrm{K} \ldots \mathrm{H}_{\mathrm{II}} \mathrm{XXR}_{\mathrm{II}} \ldots \mathrm{Y}$ pentad (where $\mathrm{X}$ is any residue), but with the typical Glu/Asp ${ }_{I}$ and His/Trp $\mathrm{III}_{\mathrm{II}}$ sites substituted by Gly/Ala and Ala/Val residues, respectively [31]. 

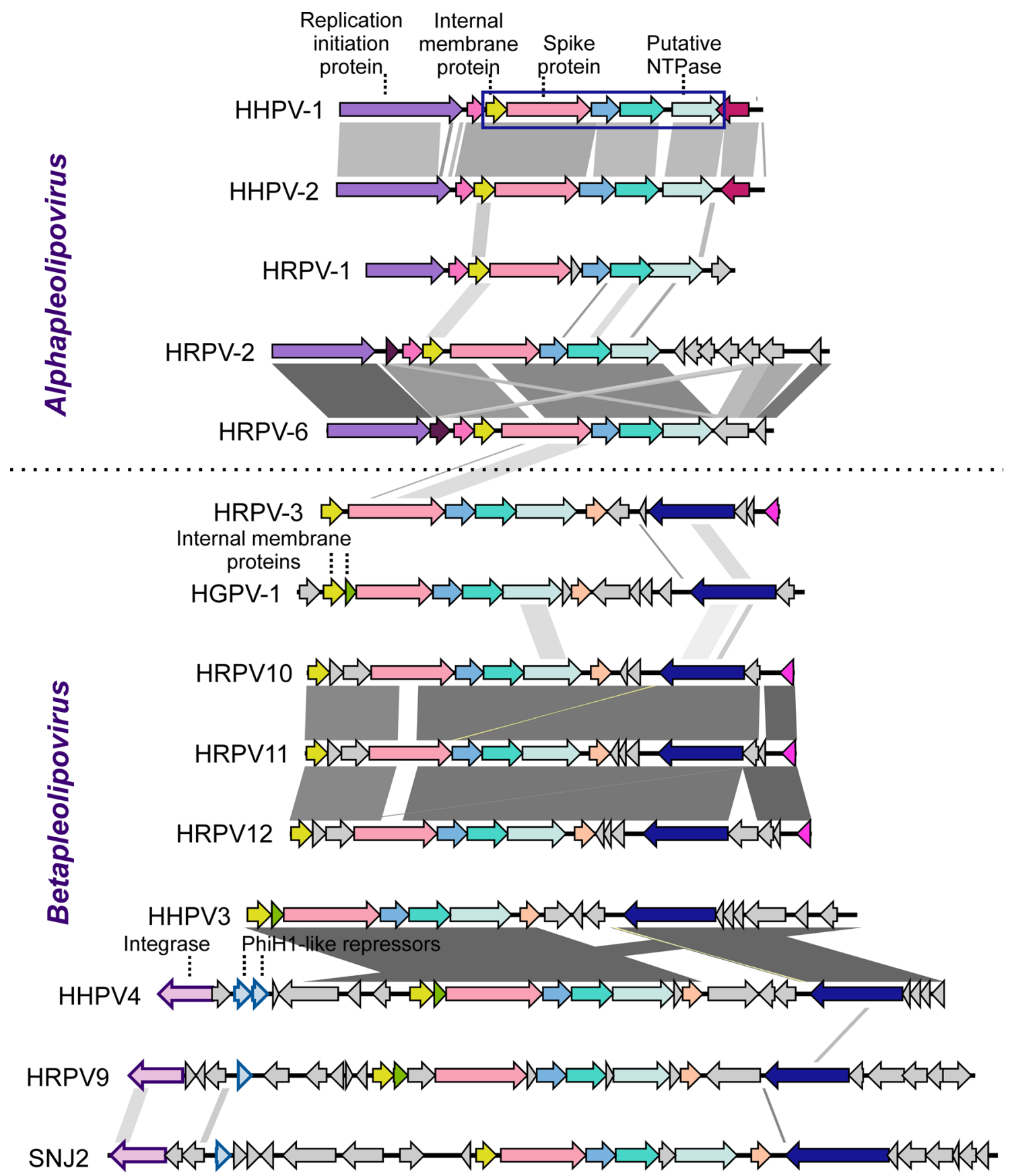

His2

\section{Gammapleolipovirus}

Fig. 3 Linear representation of the pleolipovirus genomes and the division of the viruses into the three genera in the family Pleolipoviridae shown on the left. Genes and ORFs are shown as arrows, while the shading between them represents the percentage of nucleotide sequence identity (BLASTn, max. e-value 0.001 ) from $63 \%$ to $100 \%$ (shades of grey or green for direct and inverted similarities, respec-

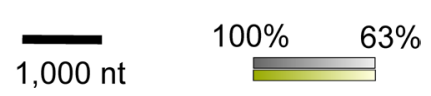

tively, see lower panel). Similar genes and ORFs are highlighted with the same colours. In Haloarcula hispanica pleomorphic virus 1 (HHPV-1), the box shows the conserved block of genes encoding the spike protein and integral membrane protein as well as ORFs with no assigned functions that are shared by all pleolipoviruses. The image was generated using Easyfig version 2.2.3 


\section{HHPV3, HHPV4, and HRPV9 survive in a saturated salt}

HHPV3 was isolated from the solar saltern of Samut Sakhon, Thailand, on Haloarcula hispanica [3]. HHPV4 and HRPV9 were isolated from plaques obtained on Har. hispanica and Halorubrum sp. SS5-4 lawns, respectively, after the addition of the other archaeal culture supernatant $[2,5]$ (Table 1). Virus particles efficiently adsorb to their host cells with adsorption rate constants of $2.4 \times 10^{-12}, 1.7 \times 10^{-12}$, and $8.5 \times 10^{-11} \mathrm{ml} / \mathrm{min}$ (measured during the first $30 \mathrm{~min}$ of infection) for HHPV3, HHPV4, and HRPV9, respectively. After $\sim 2 \mathrm{~h}$ of infection, $65-90 \%$ of the particles are bound to cell surfaces. Like other pleolipoviruses, infection with these viruses results in the retardation of the host's growth $[4,5,9]$.

HHPV 3 and HHPV4 infectivity drops in the absence of $\mathrm{NaCl}$ or $\mathrm{CaCl}_{2}[5,9]$, while for HRPV9, only the absence of $\mathrm{NaCl}$ is critical [4]. HHPV3 and HHPV4 show a pHdependent drop of infectivity at 1-1.5 $\mathrm{M} \mathrm{NaCl}[5,9]$, and HRPV9 infectivity is stable over a wide range of $\mathrm{NaCl}$ concentrations (0.5-5 M) [4]. HHPV3, HHPV4, and HRPV9, as well as betapleolipoviruses HRPV-3 and HGPV-1, stay infectious even in NaCl-saturated solutions [4, 5, 9], which may be highly beneficial for their survival in hypersaline environments.

The HHPV3, HHPV4, and HRPV9 virus particles are spherical or slightly pleomorphic with a diameter of 50-60 nm [4, 5, 9]. Like HGPV-1, the viruses HHPV3, HHPV4, and HRPV9 have one spike protein and two membrane protein types $[4,5,9,23,28]$. Lipid profiles of these viruses resemble those of their hosts, implying non-selective lipid acquisition [4, 5, 9]. The major lipids in HHPV3 and HHPV4 are phosphatidylglycerol, phosphatidylglycerophosphate methyl ester, phosphatidylglycerosulfate, and triglycosyl glycerodiether [5, 9]. The HHPV3, HHPV4, and HRPV9 genomes are circular dsDNA molecules of 11,648, 15,010 , and 16,159 bp in length, respectively, with a GC content of $55-60 \%[4,5,9]$. Single-stranded interruptions have been suggested in genomes of HHPV 3 and HHPV4 $[5,9]$. The two viruses have identical genome regions, covering $\sim 65 \%$ and $\sim 84 \%$ of the whole genome sequences in HHPV4 and HHPV3, respectively. These identical regions include the conserved block of betapleolipoviral genes: all characteristic betapleolipoviral ORFs are 100\% identical in HHPV3 and HHPV4, except that HHPV4 ORF16 and HHPV3 ORF7 share $73 \%$ nucleotide sequence identity. The case of HHPV 3 and HHPV4 highlights flexibility in pleolipoviral virion assembly, as the larger genome of HHPV4 is packed into vesicles made up of the same major structural components as in HHPV3, which has a smaller genome $[5,9]$. Notably, the HHPV4 genome contains a block of an integrase and two phiH1-like repressor genes, which is absent in HHPV3 [5]. The integrase and phiH1-like repressor genes are also found in the HRPV9 genome [4]. The HHPV4 integrase belongs to the SNJ2-type family, while HRPV9 integrase differs, with a Met substitution at the His/ $\operatorname{Trp}_{\text {III }}$ site, where SNJ2-type integrases typically have Ala/ Val (see above) [4, 31]. It is unclear whether HHPV4 and HRPV9 integrases are functional or defective [4]. Phylogenomic trees inferred using the Genome-BLAST Distance Phylogeny (GBDP) method suggest that HHPV3, HHPV4, and HRPV9 cluster with SNJ2 and other betapleolipoviruses [4].

\section{Closely related betapleolipoviruses HRPV10, HRPV11, and HRPV12 from Africa}

The most recently discovered viruses, HRPV10, HRPV11, and HRPV12, were isolated from the saline Lake Retba, close to Dakar, Senegal, on Halorubrum strains originating from the same location [19]. The viruses produce hazy plaques, typical for pleolipoviruses, suggesting a non-lytic virus life cycle. Out of 19 autochthonous Halorubrum strains from Lake Retba that were tested, HRPV11 was found to infect four, while HRPV10 and HRPV12 were capable of infecting only two strains. Out of 51 Halorubrum strains from other geographical locations, HRPV11 could infect one strain from Eilat, while the other two viruses had no additional hosts [19]. Thus, these three viruses have narrow and distinct host ranges with a preference for autochthonous strains (Table 1).

HRPV10, HRPV11, and HRPV12 form tailless round virus particles of $\sim 55 \mathrm{~nm}$ in diameter, having distinct protein profiles with two major structural proteins. The lipid content seems to be identical in the three viruses, and a nonselective mode of lipid acquisition is suggested. The virus genomes are circular dsDNA molecules with single-stranded regions. The total genome length of HRPV10, HRPV11, and HRPV12, is 9,296, 9,368, and 9,944 bp, respectively, and their GC content is $55.5 \%$. The genomes contain 13-16 ORFs, which are arranged in two putative operons. The three viruses display close genetic similarity, sharing 92-95\% overall nucleotide sequence identity, but they are genetically distant from all other pleolipoviruses. In addition to the conserved core genes found in all pleolipoviruses, HRPV10, HRPV11, and HRPV12 have ORFs that are specific to all known betapleolipoviruses (homologues of HRPV10 ORF8 and ORF11) as well as the ORFs conserved in some betapleolipoviruses (HRPV10 ORF10 and ORF13). Phylogenomic trees constructed using the GBDP method show the clustering of these three viruses with HRPV-3, HGPV-1, HHPV3, and SNJ2, i.e., the members of the genus Betapleolipovirus [19]. 


\section{Conclusions}

Concerning virion morphology, pleolipoviruses resemble bacteriophages belonging to the family Plasmaviridae [15], although they share no common genes. Deep evolutionary relationships have been proposed between pleolipoviruses and mobile genetic elements, including plasmids enclosed in membrane vesicles $[13,14]$. However, the evolutionary pathways are still unclear for this virus group. Having been neglected until 2009, pleomorphic archaeal viruses now seem to be extremely abundant in hypersaline environments all over the planet. The identification of numerous related putative proviruses in the genomes of halophilic archaea that inhabit various locations, including the deep sea [18], also highlights the abundance of pleolipoviruses and related elements. The family Pleolipoviridae is expanding fast, and we anticipate that future studies will reveal many more of its members, and/or closely related viruses.

Acknowledgements Open access funding provided by University of Helsinki including Helsinki University Central Hospital. This work was supported by the University of Helsinki and Academy of Finland funding for FINStruct (Instruct-FI), part of Biocenter Finland and InstructERIC, Ella and Georg Ehrnrooth Foundation (T. D.), and Maj and Tor Nessling Foundation (T. D.)

\section{Compliance with ethical standards}

Conflict of interest The authors declare that they have no conflict of interest.

Open Access This article is licensed under a Creative Commons Attribution 4.0 International License, which permits use, sharing, adaptation, distribution and reproduction in any medium or format, as long as you give appropriate credit to the original author(s) and the source, provide a link to the Creative Commons licence, and indicate if changes were made. The images or other third party material in this article are included in the article's Creative Commons licence, unless indicated otherwise in a credit line to the material. If material is not included in the article's Creative Commons licence and your intended use is not permitted by statutory regulation or exceeds the permitted use, you will need to obtain permission directly from the copyright holder. To view a copy of this licence, visit http://creativecommons.org/licenses/by/4.0/.

\section{References}

1. Atanasova NS, Roine E, Oren A, Bamford DH, Oksanen HM (2012) Global network of specific virus-host interactions in hypersaline environments. Environ Microbiol 14:426-440

2. Atanasova NS, Pietilä MK, Oksanen HM (2013) Diverse antimicrobial interactions of halophilic archaea and bacteria extend over geographical distances and cross the domain barrier. Microbiol Open 2:811-825

3. Atanasova NS, Demina TA, Buivydas A, Bamford DH, Oksanen HM (2015) Archaeal viruses multiply: temporal screening in a solar saltern. Viruses 7:1902-1926

4. Atanasova NS, Demina TA, Krishnam Rajan Shanthi SNV, Oksanen HM, Bamford DH (2018) Extremely halophilic pleomorphic archaeal virus HRPV9 extends the diversity of pleolipoviruses with integrases. Res Microbiol 169:500-504

5. Atanasova NS, Heiniö CH, Demina TA, Bamford DH, Oksanen HM (2018) The unexplored diversity of pleolipoviruses: the surprising case of two viruses with identical major structural modules. Genes 9:E131

6. Bamford DH, Pietilä MK, Roine E, Atanasova NS, Dienstbier A, Oksanen HM, ICTV Report Consortium (2017) ICTV virus taxonomy profile: Pleolipoviridae. J Gen Virol 98:2916-2917

7. Bath C, Cukalac T, Porter K, Dyall-Smith ML (2006) His1 and His2 are distantly related, spindle-shaped haloviruses belonging to the novel virus group, Salterprovirus. Virology 350:228-239

8. Chen S, Wang C, Xu JP, Yang ZL (2014) Molecular characterization of pHRDV1, a new virus-like mobile genetic element closely related to pleomorphic viruses in haloarchaea. Extremophiles 18:195-206

9. Demina TA, Atanasova NS, Pietilä MK, Oksanen HM, Bamford DH (2016) Vesicle-like virion of Haloarcula hispanica pleomorphic virus 3 preserves high infectivity in saturated salt. Virology 499:40-51

10. Demina TA, Krupovic M, Oksanen HM (2020) Create seven new species in the genus Betapleolipovirus, family Pleolipoviridae. International Committee on Taxonomy of Viruses, Taxonomic proposal 2019. https://talk.ictvonline.org/files/proposals/taxon omy_proposals_prokaryote $1 / \mathrm{m} /$ bact04/9102. Accessed 31 Oct 2019

11. Dyall-Smith ML, Pfeiffer F, Klee K, Palm P, Gross K, Schuster SC, Rampp M, Oesterhelt D (2011) Haloquadratum walsbyi: limited diversity in a global pond. PLoS One 6:e20968

12. El Omari K, Li S, Kotecha A, Walter TS, Bignon EA, Harlos K, Somerharju P, De Haas F, Clare DK, Molin M, Hurtado F, Li M, Grimes JM, Bamford DH, Tischler ND, Huiskonen JT, Stuart DI, Roine E (2019) The structure of a prokaryotic viral envelope protein expands the landscape of membrane fusion proteins. Nat Commun 10:846

13. Erdmann S, Tschitschko B, Zhong L, Raftery MJ, Cavicchioli R (2017) A plasmid from an Antarctic haloarchaeon uses specialized membrane vesicles to disseminate and infect plasmid-free cells. Nat Microbiol 2:1446-1455

14. Krupovic M, Cvirkaite-Krupovic V, Iranzo J, Prangishvili D, Koonin EV (2018) Viruses of archaea: structural, functional, environmental and evolutionary genomics. Virus Res 244:181-193

15. Krupovic M, ICTV Report Consortium (2018) ICTV virus taxonomy profile: Plasmaviridae. J Gen Virol 99:617-618

16. Li M, Wang R, Zhao D, Xiang H (2014) Adaptation of the Haloarcula hispanica CRISPR-Cas system to a purified virus strictly requires a priming process. Nucleic Acids Res 42:2483-2492

17. Liu Y, Wang J, Liu Y, Wang Y, Zhang Z, Oksanen HM, Bamford $\mathrm{DH}$, Chen X (2015) Identification and characterization of SNJ2, the first temperate pleolipovirus integrating into the genome of the SNJ1-lysogenic archaeal strain. Mol Microbiol 98:1002-1020

18. Messina E, Sorokin DY, Kublanov IV, Toshchakov S, Lopatina A, Arcadi E, Smedile F, La Spada G, La Cono V, Yakimov MM (2016) Complete genome sequence of 'Halanaeroarchaeum sulfurireducens' M27-SA2, a sulfur-reducing and acetate-oxidizing haloarchaeon from the deep-sea hypersaline anoxic lake Medee. Stand Genom Sci 11:35

19. Mizuno CM, Prajapati B, Lucas-Staat S, Sime-Ngando T, Forterre P, Bamford DH, Prangishvili D, Krupovic M, Oksanen HM (2019) Novel haloarchaeal viruses from Lake Retba infecting Haloferax and Halorubrum species. Environ Microbiol 21:2129-2147

20. Oksanen HM, Krupovic, M. (2020) Rename eight species in the family Pleolipoviridae. International Committee on Taxonomy of Viruses, Taxonomical proposal 2019. https://talk.ictvonline.org/ files/proposals/taxonomy_proposals_prokaryote $1 / \mathrm{m} /$ bact $04 / 9100$. Accessed 31 Oct 2019 
21. Pietilä MK, Roine E, Paulin L, Kalkkinen N, Bamford DH (2009) An ssDNA virus infecting archaea: a new lineage of viruses with a membrane envelope. Mol Microbiol 72:307-319

22. Pietilä MK, Laurinavicius S, Sund J, Roine E, Bamford DH (2010) The single-stranded DNA genome of novel archaeal virus Halorubrum pleomorphic virus 1 is enclosed in the envelope decorated with glycoprotein spikes. J Virol 84:788-798

23. Pietilä MK, Atanasova NS, Manole V, Liljeroos L, Butcher SJ, Oksanen HM, Bamford DH (2012) Virion architecture unifies globally distributed pleolipoviruses infecting halophilic archaea. J Virol 86:5067-5079

24. Pietilä MK, Roine E, Sencilo A, Bamford DH, Oksanen HM (2016) Pleolipoviridae, a newly proposed family comprising archaeal pleomorphic viruses with single-stranded or doublestranded DNA genomes. Arch Virol 161:249-256

25. Porter K, Dyall-Smith ML (2008) Transfection of haloarchaea by the DNAs of spindle and round haloviruses and the use of transposon mutagenesis to identify non-essential regions. Mol Microbiol 70:1236-1245

26. Prangishvili D, Bamford DH, Forterre P, Iranzo J, Koonin EV, Krupovic M (2017) The enigmatic archaeal virosphere. Nat Rev Microbiol 15:724-739
27. Roine E, Kukkaro P, Paulin L, Laurinavicius S, Domanska A, Somerharju P, Bamford DH (2010) New, closely related haloarchaeal viral elements with different nucleic acid types. J Virol 84:3682-3689

28. Sencilo A, Paulin L, Kellner S, Helm M, Roine E (2012) Related haloarchaeal pleomorphic viruses contain different genome types. Nucleic Acids Res 40:5523-5534

29. Stolt P, Zillig W (1992) In vivo studies on the effects of immunity genes on early lytic transcription in the Halobacterium salinarium phage phi H. Mol Gen Genet 235:197-204

30. Svirskaite J, Oksanen HM, Daugelavicius R, Bamford DH (2016) Monitoring physiological changes in haloarchaeal cell during virus release. Viruses 8:59

31. Wang J, Liu Y, Liu Y, Du K, Xu S, Wang Y, Krupovic M, Chen $X$ (2018) A novel family of tyrosine integrases encoded by the temperate pleolipovirus SNJ2. Nucleic Acids Res 46:2521-2536

Publisher's Note Springer Nature remains neutral with regard to jurisdictional claims in published maps and institutional affiliations. 